\title{
A Proposed Method to Minimize the Flow Time Average and the Due Date Average in Sequence Problem
}

\author{
Ghazwan H. Mahmoud \\ College of Computer Science and Mathematics \\ University of Mosul, Mosul, Iraq
}

Received on: 20/07/2009

Accepted on: 28/12/2009

\begin{abstract}
The problem tackled in this research is to propose a method to minimize the flow time average and the due date average to resolve the sequence problem. Assuming that we have a set of jobs, and every job has processing time and due date required to running by method that satisfy balance and minimize the flow time average and the due date average. The research presents the methods used to solve the former. The paper compares the new method with the Critical ratio (CR) method on a set of data generated by simulation by system (MATLAB). The results show a high efficiency and ease of use.
\end{abstract}

Keywords: flow time average, due date average, sequences, Critical ratio (CR) .

$$
\begin{aligned}
& \text { طريقة مقترحة لتقليل معدل الانسيابية ومعدل التأخير في مسائل المتتابعات } \\
& \text { غزوان هاني محمود } \\
& \text { كلية علوم الحاسبات والرياضيات } \\
& \text { جامعة الموصل }
\end{aligned}
$$

تاريخ قبول البحث: 2009/12/28

تاريخ استلام البحث: 2009/7/20

\section{الملخص}

أن المسالة التي يتتاولها هذا البحث هي اقتراح طريقة لتقليل معدل الانسيابية ومعدل التأخير لحل مسائل

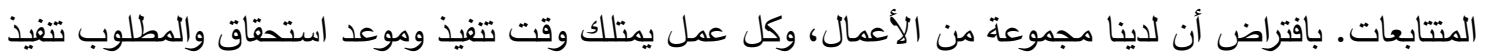

تلك الأعمال بالطريقة التي تحقق الاتزان وتقلل من معدل انسيابية الأعمال ومعدل التأخير • وقد تم في هذا البحث

عرض الطرق السابقة لحل مثل هذه المسائل وتوضيحها، وكذلك عرض الطريقة المقترحة وتم مقارنتها مع طريقة

Critical Ratio (CR)

$$
\text { كفاءة عالية وسهولة بالاستخدام. }
$$

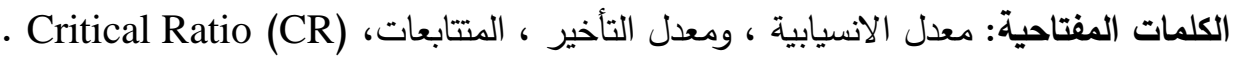

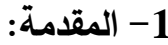

تعتبر إدارة المشاريع من الأعمال المهمة لأي مؤسسة للسيطرة على وقت إنهاء العمل والإنتاج والسيطرة

على التأخير، وتهدف مشاكل التتابع إلى تحقيق اقل وقت مدكن أو اقل كلفة ممكنة أو أداء مدكن لعمليتين أو أكثر 
(المستهلكين اللذين تقدم لهم ألخدمه ) يراد إنجازها، مع وجود واحد أو أكثر من الإمكانيات المتاحة (مراكز عمل مخصصة) لإنجاز معالجة تلك الإعمال.

ولتتفيذ هذه الأعمال وفق تسلسل معين تظهر مشاكل التتابع في مراكز الأعمال حيث تتدرج من الأبسط

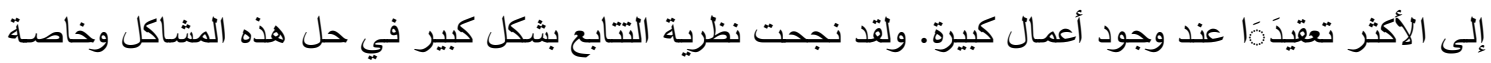
التي تعمل حسب الطلب

وفي ضوء ما تقدم يمكن تعريف التتابع بأنه إيجاد التسلسل الأمثل للمراحل الضرورية لإنجاز مشروع معين بالثكل الذي يحقق تتفيذ مجموعة من الأعمال بأقل وقت أو جهد ونتيجة لذلك يكون بأقل كلفة مدكنة.

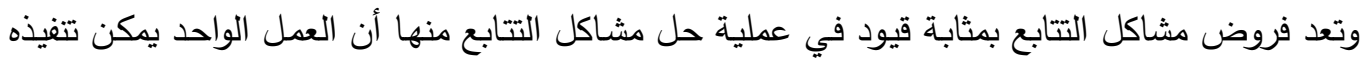
في مركز عمل واحد وعند البدء بتتفيذه في إحدى مراكز العمل لابد أن يستمر حتى إتمامهه. وتثترط مشاكل التتابع

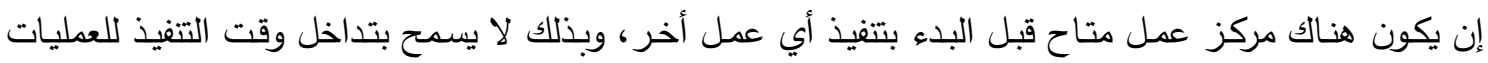

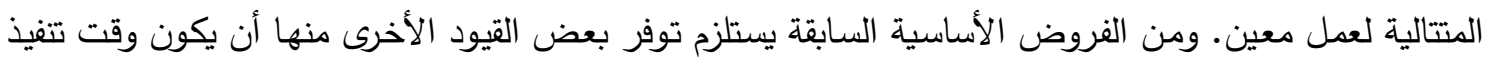
أي عمل في أي مركز عمل قابلا للقياس.

إن الهدف الأساسي مـن هذا البحث هو عر عرض بعض ابل الطرق (القواعد) المستخدمة في حل مسـائل

المتتابعات لتقليل معدل الانسيابية للأعمال ومعدل التأخير ومعدل عدد الأعمال في النظام، وذلك من خـلال استخدام قواعد الأسبقية في جدولة مجموعة من الأعمال. وتم في هذا البحث التوصل لطريقة جديدة تمكننا من

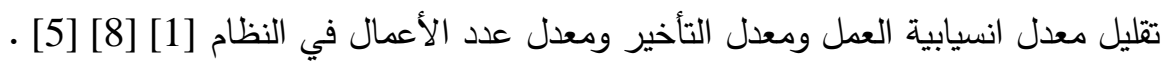
2يمكن تقسيم المفاهيم الأساسية إلى قسمين وهما [3] [4]:

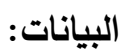

:Sequencing المتتابعات 1-2 تحديد العمل الذي سيتم تتفيذه من بين مجموعة من الأعمال في مركز إدارة المشروع. 2-2-2 وقت التنفيذ (processing time): هو الوقت الذي يستغرقه كل عمل لتتفيذه. 3-2 تاريخ الاستحقاق (due date):

هو الوقت اللازم الذي يجب فيه تسليم العمل بصورته النهائية إلى صاحب العمل (المستهلك). ويحدد هذا الوقت من قبل المستهلك.

4-2-2 وقت إنهاء العمل(make span):

هو الزمن الكلي اللازم لإنهاء تتفيذ مجموعة من الأعمال ويمكن حسابه من العلاقة التالية: وقت إنهاء العمل = مجموع أوقات التتفيذ لجميع الأعمال make span $=$ sum of processing times 
المعاييز:

:5low time) -5-2 (نسيابية العمل (flow)

هو الوقت اللازم من بدأ تتفيذ الأعمال في مركز العمل لإتمام العمل, أو هو عبارة عن التكرار المتجمع الصاعد لأوقات التتفيذ ويمكن حساب معدل الانسيابية حسب الصيغة التالية:

معدل انسيابية العمل = مجموع أوقات انسيابية العمل/ عدد الأعمال

average flow time $=$ sum of flow times $/$ no. of jobs

6-2 وقت التأخير (Tardy time):

هو الاختلاف الموجب بين انسيابية العمل وتاريخ الاستحقاق للأعمال حيث تهمل فيها القيم ألسالبة وتصبح صفرا ويحسب بالصيغة التالية: وقت التأخير = انسيابية العمل - تأريخ الاستحقاق tardy = flow time-due date

أما معدل وقت التأخير فيحسب بالصيغة التالية: معدل التأخير = مجموع التأخير /عدد الأعمال

average of tardiness $=$ sum tardy $/$ no. of jobs

7-2- معدل عدد الأعمال في النظام ( Average number of jobs in system): هو مجموع أوقات انسيابية العمل مقسومة على مجموع أوقات إنهاء العمل الكلي لجميع الأعمال.ويمكن توضيحه بالصيغة التالية:

معدل عدد الأعمال بالنظام = مجموع انسيابية العمل / وقت إنهاء العمل. average no. of jobs in system = sum of flow times / make span

3- قواعد الأسبقية Priority Rules):

يشير تتابع الأعمال إلى ترتيب الأعمال بالثكل الذي يتم بموجبة معالجة الأعمال في مراكز العمل، وان

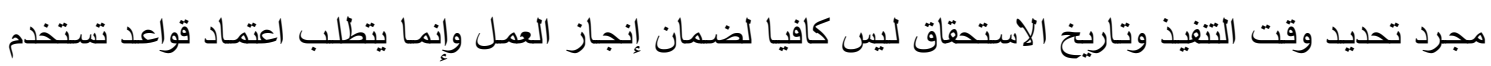
لتحديد أسبقيات تتابع الأعمال على مراكز العمل ومن هذه القواعد نذكر ما يلي [1] [9: [9]:

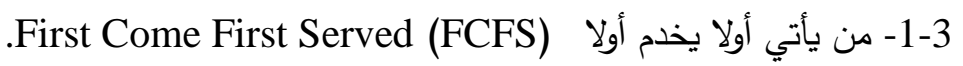

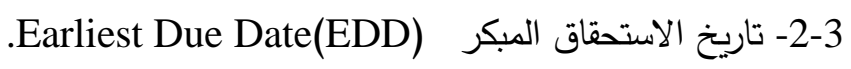

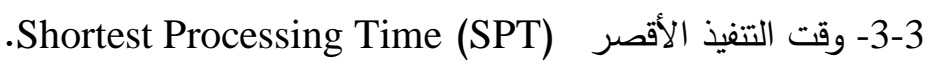

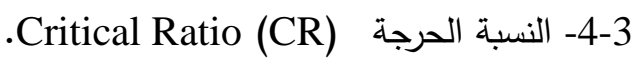

ولتوضيح طريقة عمل كل طريقة سيتم اخذ مثال توضيحي ومن خلاله نجد معدل انسيابية الأعمال ومعدل التأخير ووقت إنهاء التتفيذ ومعدل عدد الأعمال بالنظام:- 
1-3- من يأتي أولا يخدم أولا First Come First Served (FCFS)

تقترض هذه الطريقة إن الأسبقية في إنجاز العمل تكون للعمل الذي يصل أولا لككان الخدمة، وبذلك لن

تكون هنالك أسبقية أو تفضيل أي عمل على عمل أخر ، ولتوضيح ذلك نأخذ المثال التالي [1] :-

\begin{tabular}{|c|c|c|}
\hline jobs & processing time & Due date \\
\hline 1 & 4 & 9 \\
\hline 2 & 9 & 14 \\
\hline 3 & 2 & 7 \\
\hline 4 & 5 & 5 \\
\hline 5 & 7 & 11 \\
\hline
\end{tabular}

الحل: نجد جدول الانسيابية وذلك عن طريق التكرار المتجمع الصاعد لجدول أوقات التنفيذ، ثم نجد جدول أوقات التأخير وذلك عن طريق طرح جدول تاريخ الاستحقاق من جدول أوقات الانسيابية ويصبح لدينا جدول الحل الكامل

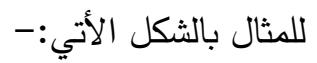

\begin{tabular}{|c|c|c|c|c|}
\hline jobs & $\begin{array}{c}\text { processing } \\
\text { time }\end{array}$ & due date & flow time & tardy \\
\hline 1 & 4 & 9 & 4 & 0 \\
\hline 2 & 9 & 14 & 13 & 0 \\
\hline 3 & 2 & 7 & 15 & 8 \\
\hline 4 & 5 & 5 & 20 & 15 \\
\hline 5 & 7 & 11 & 27 & 16 \\
\hline Total & 27 & 46 & 79 & 39 \\
\hline
\end{tabular}

$$
\text { 1- معدل انسيابية العمل = مجموع أوقات الانسيابية / عدد الأعمال }
$$

average flow time $=79$ / $5=15.8$

2- معدل التأخير = مجموع أوقات التأخير/ / عدد الأعمال

Average tardy $=39 / 5=7.8$

$$
\text { 3- وقت إنهاء العمل الكلي = مجموع أوقات التنفيذ }
$$

make span $=27$

4- معدل عدد الأعمال في النظام = مجموع أوقات الانسيابية / وقت إنهاء العمل

average no. Jobs in system = 79 / $27=2.95$

2-3-3 تاريخ الاستحقاق المبكر Earliest Due Date (EDD):

تهدف هذه الطريقة إلى تقليل عدد الأعمال المتأخرة حيث تقوم على أساس تجهيز العمل وإنجازه متى ما

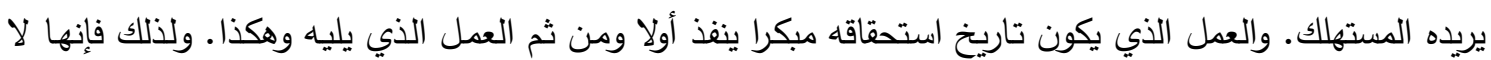

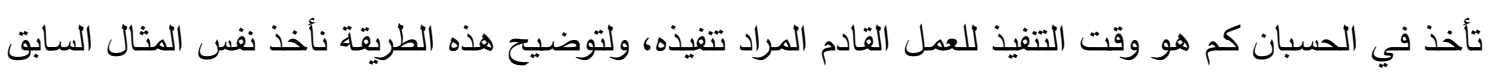
وكالاتي [2] [6]:-

\begin{tabular}{|c|c|c|}
\hline jobs & Processing time & Due date \\
\hline 1 & 4 & 9 \\
\hline
\end{tabular}




\begin{tabular}{|c|c|c|}
\hline 2 & 9 & 14 \\
\hline 3 & 2 & 7 \\
\hline 4 & 5 & 5 \\
\hline 5 & 7 & 11 \\
\hline
\end{tabular}

الحل: بعد ترتيب الأعمال حسب تاريخ الاستحقاق المبكر يصبح لدينا الجدول كالآتي:-

\begin{tabular}{|c|c|c|}
\hline jobs & processing time & Due date \\
\hline 4 & 5 & 5 \\
\hline 3 & 2 & 7 \\
\hline 1 & 4 & 9 \\
\hline 5 & 7 & 11 \\
\hline 2 & 9 & 14 \\
\hline
\end{tabular}

نجد جدول الانسيابية وذلك عن طريق التكرار المتجمع الصاعد لجدول أوقات التنفيذ، ثم نجد جدول أوقات التأخير وذلك عن طريق طرح جدول تاريخ الاستحقاق من جدول أوقات الانسيابية ويصبح لدينا جدول الحل الكامل للمثال

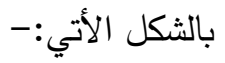

\begin{tabular}{|c|c|c|c|c|}
\hline jobs & $\begin{array}{c}\text { processing } \\
\text { time }\end{array}$ & Due date & Flow time & tardy \\
\hline 4 & 5 & 5 & 5 & 0 \\
\hline 3 & 2 & 7 & 7 & 0 \\
\hline 1 & 4 & 9 & 11 & 2 \\
\hline 5 & 7 & 11 & 18 & 7 \\
\hline 2 & 9 & 14 & 27 & 13 \\
\hline Total & 27 & 46 & 68 & 22 \\
\hline
\end{tabular}

1- معدل انسيابية العمل = مجموع أوقات الانسيابية / عدد الأعمال average flow time $=68 / 5=13.6$

2- معدل التأخير = مجموع أوقات التأخير / عدد الأعمال

average tardy $=22 / 5=4.4$

3- وقت إنهاء العمل الكلي = مجموع أوقات التنفيذ

make span $=27$

4- معدل الأعمال في النظام = مجموع أوقات الانسيابية / وقت إنهاء العمل 68 إن

average no. Jobs in system $=68 / 27=2.52$

Shortest Processing Time (SPT) وقت التنفيذ الأقصر

يتم وفق هذه الطريقة معالجة العمل الذي يمتلك وقت تتفيذ اقصر ، أي أن العمل الذي وقت تتفيذه اقل يخدم أولا لذلك فان الأعمال التي تتطلب أوقات تتفيذ أطول قد تعالج بصوره متأخرة جدا لأنها تبقى في الانتظار

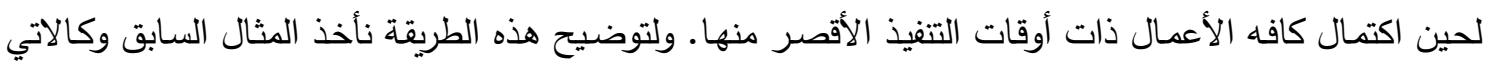

\begin{tabular}{|c|c|c|}
\hline jobs & processing time & Due date \\
\hline 1 & 4 & 9 \\
\hline
\end{tabular}




\begin{tabular}{|c|c|c|}
\hline 2 & 9 & 14 \\
\hline 3 & 2 & 7 \\
\hline 4 & 5 & 5 \\
\hline 5 & 7 & 11 \\
\hline
\end{tabular}

الحل:-

بعد ترتيب الأعمال حسب أوقات التتفيذ الأقصر يصبح لدينا الجدول كالأتي:-

\begin{tabular}{|c|c|c|}
\hline jobs & processing time & Due date \\
\hline 3 & 2 & 7 \\
\hline 2 & 4 & 9 \\
\hline 4 & 5 & 5 \\
\hline 5 & 7 & 11 \\
\hline 1 & 9 & 14 \\
\hline
\end{tabular}

نجد جدول الانسيابية وذلك عن طريق التكرار المتجمع الصاعد لجدول أوقات التتفيذ، ثم نجد جدول أوقات التأخير وذلك عن طريق طرح جدول تاريخ الاستحقاق من جدول أوقات الانسيابية ويصبح لدينا جدول الحل الكامل للمثال

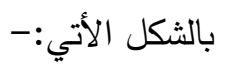

\begin{tabular}{|c|c|c|c|c|}
\hline jobs & $\begin{array}{c}\text { processing } \\
\text { time }\end{array}$ & Due date & flow time & tardy \\
\hline 3 & 2 & 7 & 2 & 0 \\
\hline 2 & 4 & 9 & 6 & 0 \\
\hline 4 & 5 & 5 & 11 & 6 \\
\hline 5 & 7 & 11 & 18 & 7 \\
\hline 1 & 9 & 14 & 27 & 13 \\
\hline Total & 27 & 46 & 64 & 26 \\
\hline
\end{tabular}

1- معدل انسيابية العمل = مجموع أوقات الانسيابية / عدد الأعمال average flow time $=64 / 5=12.8$

$$
\begin{aligned}
& \text { 2- معدل التأخير = مجموع أوقات التأخير / عدد الأعمال } \\
& \text { average tardy }=26 / 5=5.2
\end{aligned}
$$

3- وقت إنهاء العمل الكلي = مجموع أوقات التنفيذ

make span $=27$

4- معدل الأعمال في النظام = مجموع أوقات الانسيابية / وقت إنهاء العمل

average no. Jobs in system $=64 / 27=2.37$

\section{Critical Ratio(CR) النسبة الحرجة:-3-3}

تعتبر من أفضل واهم الطرق المستخدمة لحل مسالة المتتابعات لكونها تحقق الاتزان بين وقت التتفيذ وموعد الاستحقاق. وتمثل هذه النسبة التوازن بين طريقة (SPT) وطريقة (EDD) وتعتمد على اقصر زمن متبقي

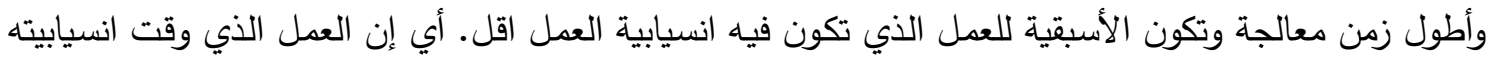

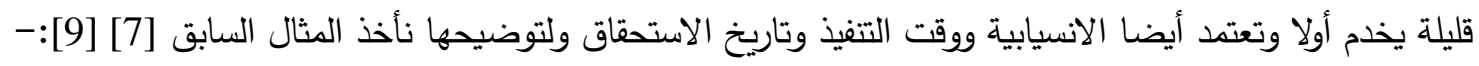




\begin{tabular}{|c|c|c|}
\hline jobs & Processing time & Due date \\
\hline 1 & 4 & 9 \\
\hline 2 & 9 & 14 \\
\hline 3 & 2 & 7 \\
\hline 4 & 5 & 5 \\
\hline 5 & 7 & 11 \\
\hline
\end{tabular}

الخطوة الأولى:- نقوم بحساب النسبة الحرجة (CR)حسب الصيغة التالية:critical ratio $=($ due date - flow time $) /$ processing time النسبة الحرجة = (تاريخ الاستحقاق- انسيابية العمل) / وقت التنفيذ ثم نأخذ اقل نسبة ويمكن التوضيح كالأتي:-

\begin{tabular}{|c|c|c|c|}
\hline jobs & $\begin{array}{c}\text { processing } \\
\text { time }\end{array}$ & due date & critical ratio \\
\hline 1 & 4 & 9 & $(9-0) / 4=2.25$ \\
\hline 2 & 9 & 14 & $(14-0) / 9=1.56$ \\
\hline 3 & 2 & 7 & $(7-0) / 2=3.50$ \\
\hline 4 & 5 & 5 & $(5-0) / 5=1.00$ \\
\hline 5 & 7 & 11 & $(11-0) / 7=1.57$ \\
\hline
\end{tabular}

نلاحظ أن انسيابية العمل في الخطوة الأولى تساوي (0) وذلك لكون انه لم يتم تنفيذ أي عمل. وبتطبيق قانون

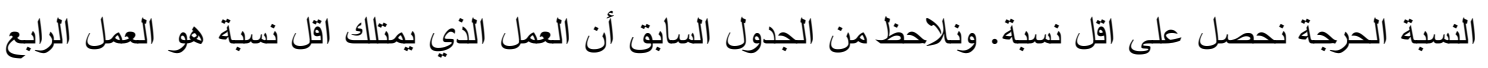
لذلك سيتم تتفيذه أولا. الخطوة الثانية:-

\begin{tabular}{|c|c|c|c|}
\hline jobs & $\begin{array}{c}\text { processing } \\
\text { time }\end{array}$ & due date & critical ratio \\
\hline 1 & 4 & 9 & $(9-5) / 4=1.00$ \\
\hline 2 & 9 & 14 & $(14-5) / 9=1.00$ \\
\hline 3 & 2 & 7 & $(7-5) / 2=1.00$ \\
\hline 5 & 7 & 11 & $(11-5) / 7=0.86$ \\
\hline
\end{tabular}

نلاحظ أن انسيابية العمل في الخطوة الثانية تساوي (5) وذلك لكون انه تم تتفيذ العمل الرابع والذي وقت تتفيذه يساوي (5). وبتطبيق قانون النسبة الحرجة نحصل على اقل نسبة. ونلاحظ من الجدول السابق أن العمل الذي يمتلك اقل نسبة هو العمل الخامس لذلك سيتم تتفيذه ثانيا. الخطوة الثالثة:- - الثان

\begin{tabular}{|c|c|c|c|}
\hline jobs & processing time & due date & critical ratio \\
\hline 1 & 4 & 9 & $(9-12) / 4=-0.75$ \\
\hline 2 & 9 & 14 & $(14-12) / 9=0.22$ \\
\hline 3 & 2 & 7 & $(7-12) / 2=-2.50$ \\
\hline
\end{tabular}


نلاحظ أن انسيابية العمل في الخطوة الثالثة تساوي (12) وذلك لكون انه تم تتفيذ العمل الرابع والخامس واللذان يكون وقت تتفيذهما يسـاوي (12=7+5). وبتطبيق قانون النسبة الحرجـة نحصل على اقل نسبة. ونلاحظ من الجدول السابق أن العمل الذي يمتلك اقل نسبة هو العمل الثالث لذلك سيتم تتفيذه ثالثا.

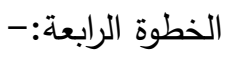

\begin{tabular}{|c|c|c|c|}
\hline jobs & processing time & due date & critical ratio \\
\hline 1 & 4 & 9 & $(9-14) / 4=-1.25$ \\
\hline 2 & 9 & 14 & $(14-14) / 9=0.00$ \\
\hline
\end{tabular}

نلاحظ أن انسيابية الععل في الخطوة الثالثة تساوي (14) وذلك لكون انه تم تتفيذ العمل الرابع والخامس والثالث والذين يكون وقت تتفيذهم يساوي (14=2+7+5). وبتطبيق قانون النسبة الحرجة نحصل على اقل نسبة. ونلاحظ من الجدول السابق أن العمل الذي يمتلك اقل نسبة هو العمل الأول لذلك سيتم تتفيذه رابعا. ثم بعد ذلك يتم تتفيذ العمل الأخير (العمل الثاني). وبعد إجراء الخطوات السابقة يصبح جدول الحل كالأتي:-

\begin{tabular}{|c|c|c|c|c|}
\hline jobs & $\begin{array}{c}\text { processing } \\
\text { time }\end{array}$ & Due date & flow time & tardy \\
\hline 4 & 5 & 5 & 5 & 0 \\
\hline 5 & 7 & 11 & 12 & 1 \\
\hline 3 & 2 & 7 & 14 & 7 \\
\hline 1 & 4 & 9 & 18 & 9 \\
\hline 2 & 9 & 14 & 27 & 13 \\
\hline Total & 27 & 46 & 76 & 30 \\
\hline
\end{tabular}

1- معدل انسيابية العمل = مجموع أوقات الانسيابية / عدد الأعمال average flow time $=76 / 5=15.2$

2- معدل التأخير = مجموع أوقات التأخير/ عدد الأعمال 6 / 6 / average tardy $=30 / 5=6$

3- وقت إنهاء العمل الكلي = مجموع أوقات التنفيذ make span $=27$

4- معدل عدد الأعمال في النظام = مجموع أوقات الانسيابية / وقت إنهاء العمل average no. Jobs in system $=76 / 27=2.81$

4- الطريقة المقترحة: - 2

في بداية شرح الطريقة المقترحة لابد من توضيح الأساس الذي تم الاعتماد عليه لاقتراح هذه الطريقة، إن

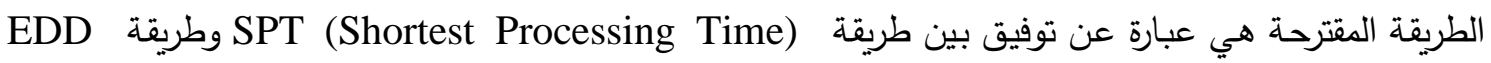
(Earliest Due Date) طريقة (EDD) فيتم تقليل معدل التأخير على حساب معدل انسيابية العمل. وكما هو معلوم فان الأمثلية تعني تحقيق الاتزان بين جميع المقاييس (المعايير). 
تتكون الطريقة المقترحة من الخطوات الآتية:

1 1- إيجاد حاصل جمع وقت التتفيذ وموعد الاستحقاق لكل عمل.

2- ترتيب المجموع الذي تم الحصول عليه في الفقرة الأولى ترتيبا تتازليا.

3- تتفيذ العمل الذي يمتلك اقل مجموع أولاثم العمل الذي يليه وهكذا.

ولتوضيح طريقة عمل الطريقة المقترحة سيتم اخذ المثال السابق

\begin{tabular}{|c|c|c|}
\hline jobs & processing time & due date \\
\hline 1 & 4 & 9 \\
\hline 2 & 9 & 14 \\
\hline 3 & 2 & 7 \\
\hline 4 & 5 & 5 \\
\hline 5 & 7 & 11 \\
\hline
\end{tabular}

الحل:-

الخطوة الأولى إيجاد حاصل جمع وقت التتفيذ وموعد الاستحقاق لكل عمل فيكون لدينا الجدول الأتي:

\begin{tabular}{|c|c|c|c|}
\hline jobs & processing time & due date & $\begin{array}{c}\text { Sum processing } \\
\text { time \& due date }\end{array}$ \\
\hline 1 & 4 & 9 & 13 \\
\hline 2 & 9 & 14 & 23 \\
\hline 3 & 2 & 7 & 9 \\
\hline 4 & 5 & 5 & 10 \\
\hline 5 & 7 & 11 & 18 \\
\hline
\end{tabular}

الخطوة الثانية ترتيب المجموع الذي تم الحصول عليه في الجدول الأول ترتيبا تتازليا فيكون لدينا الجدول الأتي:

\begin{tabular}{|c|c|c|}
\hline jobs & processing time & due date \\
\hline 3 & 2 & 7 \\
\hline 4 & 5 & 5 \\
\hline 1 & 4 & 9 \\
\hline 5 & 7 & 11 \\
\hline 2 & 9 & 14 \\
\hline
\end{tabular}

نجد جدول الانسيابية وذلك عن طريق التكرار المتجمع الصاعد لجدول أوقات التتفيذ، ثم نجد جدول أوقات التأخير وذلك عن طريق طرح جدول تاريخ الاستحقاق من جدول أوقات الانسيابية ويصبح لدينا جدول الحل الكامل للمثال

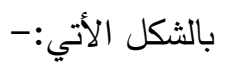

\begin{tabular}{|c|c|c|c|c|}
\hline jobs & $\begin{array}{c}\text { processing } \\
\text { time }\end{array}$ & Due date & flow time & tardy \\
\hline 3 & 2 & 7 & 2 & 0 \\
\hline 4 & 5 & 5 & 7 & 2 \\
\hline 1 & 4 & 9 & 11 & 2 \\
\hline 5 & 7 & 11 & 18 & 7 \\
\hline 2 & 9 & 14 & 27 & 13 \\
\hline
\end{tabular}




\begin{tabular}{|l|l|l|l|l|}
\hline Total & 27 & 46 & 65 & 24 \\
\hline
\end{tabular}

$$
\begin{aligned}
& \text { 1- معدل انسيابية العمل = مجموع أوقات الانسيابية / عدد الأعمال } \\
& \text { average flow time }=65 / 5=13 \\
& \text { 2- معدل التأخير = مجموع أوقات التأخير / عدد الأعمال } \\
& \text { average tardy }=24 / 5=4.8 \\
& \text { 3- وقت إنهاء العمل الكلي = مجموع أوقات التنفيذ } \\
& \text { make span }=27
\end{aligned}
$$

\begin{tabular}{|c|c|c|}
\hline الطريقة المستخدمة & معدل وقت الانسيابية & معدل وقت التأخير \\
\hline من يأتي أولا يخدم أولا & 15.8 & 7.8 \\
\hline تاريخ الاستحقاق المبكر & 13.6 & 4.4 \\
\hline وقت التنفيذ الأقصر & 12.8 & 5.2 \\
\hline النسبة الحرجة & 15.2 & 6 \\
\hline الطريقة المقترحة & 13 & 4.8 \\
\hline
\end{tabular}

average no. Jobs in system $=65 / 27=2.41$

والجدول التالي يوضـح كفاءة الطريقة المقترحة من حيث امتلاكها لمعايير (معدل وقت الانسيابية ووقت التأخير ) ذات كفاءة عالية، وتحقيقها للاتزان بين معدل وقت الانسيابية ووقت التأخير بالاعتماد على بيانات المثال السابق:

نلاحظ مـن الجدول أعـلاه أن طريقـة مـن يأتي أولا يخدم أولا ذات معـيير غير كفوءة، وطريقـة تاريخ

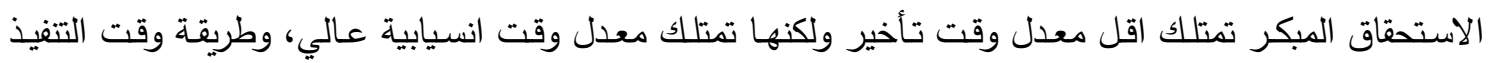
الأقصر تمتلك اقل معدل وقت انسيابية ولكنها تمتلك معدل وقت تأخير عالي، وطريقة النسبة الحرجة تحقق الاتزان بين معدل وقت الانسيابية ومعدل وقت التأخير ، أما الطريقة المقترحة فإنها تمتلك معايير ذات كفاءة عالية كذلك تحقق الاتزان بين معدل وقت الانسيابية ومعدل وقت التأخير .

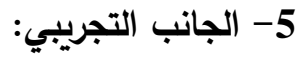

تم في هذا المبحث كتابـة برنـامج بلغـة (MATLAB) لتوليد عدد مـن الأعمـال (5,6,7,.,100) باستخدام التوزيع المنتظم وبثلاث مراحل لكل عدد، في كل مرحلة يتم تغيير فترة التوزيع المنتظم وكانت (20-1)

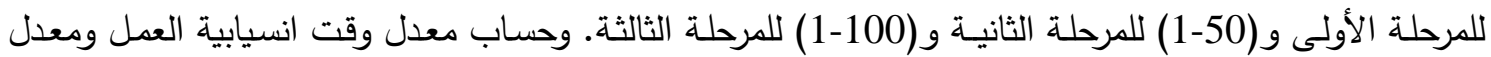

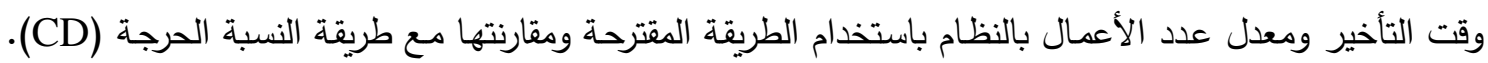
والجدول التالي يوضح عدد الأعمال والمقارنة بين الطريقتين: 


\begin{tabular}{|c|c|c|c|c|c|c|}
\hline \multirow[b]{2}{*}{ الأعمال } & \multicolumn{3}{|c|}{ الطريقة المقترحة } & \multicolumn{3}{|c|}{ طريقة النسبة الحرجة } \\
\hline & النسيابية & التأخدل & معدل عدد الأعمال & النسيابية & التأخدل & معدل عدد الأعمال \\
\hline \multirow{3}{*}{5} & 21.8000 & 11.0000 & 2.5349 & 23.0000 & 11.4000 & 2.6744 \\
\hline & 13.2000 & 5.0000 & 2.1290 & 20.0000 & 11.0000 & 3.2258 \\
\hline & 10.0000 & 3.2000 & 2.5000 & 13.2000 & 4.8000 & 3.3000 \\
\hline \multirow{3}{*}{6} & 26.3333 & 14.1667 & 2.8727 & 31.1667 & 17.8333 & 3.4000 \\
\hline & 18.0000 & 7.5000 & 2.6341 & 19.5000 & 7.8333 & 2.8537 \\
\hline & 18.6667 & 10.3333 & 2.8000 & 20.0000 & 10.8333 & 3.0000 \\
\hline \multirow{3}{*}{7} & 39.1429 & 23.4286 & 3.1860 & 44.8571 & 28.0000 & 3.6512 \\
\hline & 34.4286 & 21.5714 & 3.3014 & 37.4286 & 24.4286 & 3.5890 \\
\hline & 10.7143 & 5.2857 & 3.0000 & 13.8571 & 8.1429 & 3.8800 \\
\hline \multirow{3}{*}{8} & 29.8750 & 17.0000 & 3.1039 & 33.3750 & 19.8750 & 3.4675 \\
\hline & 28.7500 & 17.2500 & 3.5938 & 34.2500 & 22.7500 & 4.2818 \\
\hline & 26.5000 & 17.6250 & 3.8545 & 27.0000 & 17.8750 & 3.9273 \\
\hline \multirow{3}{*}{9} & 37.7778 & 26.0000 & 4.0476 & 38.6667 & 26.3333 & 4.1429 \\
\hline & 39.7778 & 28.0000 & 4.1149 & 42.6667 & 29.4444 & 4.4138 \\
\hline & 17.7778 & 10.7778 & 3.5556 & 19.4444 & 11.8889 & 3.8889 \\
\hline \multirow{3}{*}{10} & 39.2000 & 25.1000 & 3.6636 & 46.5000 & 30.0000 & 4.3458 \\
\hline & 36.2000 & 25.5000 & 4.0674 & 39.6000 & 27.7000 & 4.4494 \\
\hline & 24.2000 & 15.3000 & 4.6538 & 26.1000 & 17.1000 & 5.0192 \\
\hline \multirow{3}{*}{15} & 49.6667 & 35.8667 & 5.6015 & 56.1333 & 42.3333 & 6.3308 \\
\hline & 44.0000 & 33.4667 & 5.8407 & 49.2667 & 38.2000 & 6.5398 \\
\hline & 25.2000 & 18.4000 & 5.6418 & 26.0667 & 19.1333 & 5.8358 \\
\hline \multirow{3}{*}{20} & 88.1000 & 73.3000 & 8.0457 & 87.3500 & 72.5000 & 7.9772 \\
\hline & 58.1000 & 47.1000 & 7.9589 & 63.5500 & 52.2500 & 8.7055 \\
\hline & 53.7500 & 44.4500 & 8.3333 & 54.1500 & 44.6000 & 8.3953 \\
\hline \multirow{3}{*}{25} & 82.8000 & 68.7600 & 9.2825 & 81.9200 & 67.7200 & 9.1839 \\
\hline & 71.9600 & 60.0400 & 9.5185 & 74.2000 & 62.2400 & 9.8148 \\
\hline & 63.6000 & 54.9600 & 10.0633 & 63.6400 & 54.9200 & 10.0696 \\
\hline \multirow{3}{*}{30} & 138.7667 & 122.3333 & 11.4683 & 139.0667 & 121.9667 & 11.4931 \\
\hline & 88.1667 & 76.1667 & 11.0669 & 90.5667 & 78.5000 & 11.3682 \\
\hline & 62.8000 & 54.7667 & 11.4878 & 64.6333 & 56.4667 & 11.8232 \\
\hline \multirow{3}{*}{35} & 132.3714 & 118.3714 & 12.8694 & 132.1429 & 117.8286 & 12.8472 \\
\hline & 110.1714 & 98.6286 & 13.2966 & 111.1714 & 99.5429 & 13.4172 \\
\hline & 72.2857 & 64.4857 & 13.5294 & 74.4571 & 66.6571 & 13.9358 \\
\hline \multirow{3}{*}{40} & 159.2000 & 143.3250 & 14.3423 & 161.4000 & 145.4750 & 14.5405 \\
\hline & 127.9500 & 115.9500 & 14.8348 & 129.7000 & 117.7000 & 15.0377 \\
\hline & 86.5750 & 78.4500 & 15.4598 & 86.5750 & 78.4000 & 15.4598 \\
\hline \multirow{3}{*}{45} & 169.4444 & 154.2000 & 15.7867 & 176.1778 & 160.8667 & 16.4141 \\
\hline & 150.6444 & 138.5778 & 17.8865 & 152.0222 & 139.8000 & 18.0501 \\
\hline & 107.7333 & 98.7333 & 17.8235 & 107.5111 & 98.4889 & 17.7868 \\
\hline
\end{tabular}




\begin{tabular}{|c|c|c|c|c|c|c|}
\hline \multirow{3}{*}{50} & 179.3000 & 163.9800 & 18.3333 & 180.7200 & 165.3800 & 18.4785 \\
\cline { 2 - 8 } & 163.0200 & 150.6800 & 19.3610 & 163.8600 & 151.4400 & 19.4608 \\
\cline { 2 - 8 } & 117.8800 & 109.3800 & 19.8451 & 118.4200 & 109.8800 & 19.9360 \\
\hline \multirow{3}{*}{100} & 381.7400 & 366.7100 & 35.6766 & 384.1000 & 369.0400 & 35.8972 \\
\cline { 2 - 8 } & 272.9400 & 261.5100 & 35.8660 & 273.2400 & 261.7900 & 35.9054 \\
\cline { 2 - 8 } & 201.8900 & 193.6900 & 37.6660 & 202.1900 & 193.9900 & 37.7220 \\
\hline
\end{tabular}

في الجدول السـابق نلاحظ أن معدل وقت الانسيابية باستخدام الطريقـة المقترحة كان أفضل من طريقة النسبة الحرجة بنسبة 0.89 \%)، ومعدل وقت التأخير باستخدام الطريقة المتقرحة كان أفضل من طريقة النسبة الحرجة بنسبة 0.84 \%، أما معدل عدد الأعمال بالنظام باستخدام الطريقة المقترحة كان أفضل من طريقة النسبة الحرجة بنسبة 0.910 \%

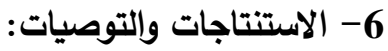
1- تم التوصل لطريقة تمتلك معايير ذات كفاءة عالية وسهولة بالاستخدام لتتفيذ مجموعة من الأعمال. 2- تم التوصل لطريقة تحقق الاتزان بين وقت التتفيذ وموعد الاستحقاق. 3- استخدام طريقة (SPT) للحصول على معدل انسيابية (flow time) قليل. 4- استخدام طريقة (EDD) للحصول على معدل التأخير (tardy) قليل. 5- استخدام الطريقة المقترحة لتحقيق الاتزان بين المعايير بدلا من طريقة (CR) ولتقليل معدل انسيابية العمل ومعدل التأخير وكذلك سهولة الحل. 


\section{المصادر}

النعيمي، محمد عبد العـال والحمداني، رفاه شهاب (1999)، "مقدمـة في بحوث العمليات"، دار وائل

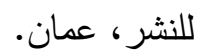

[2] Anderson, E.J., and Nyirenda, J.C., (1990), "Two New Rules to Minimize Tardiness in a Job Shop", International Journal of Production research, Vol. (28), No. (12), pp 2277-2292.

[3] Baker, K.R., (1974), "Introduction to Sequencing and Scheduling", John Wiely and Sous, Inc, New York.

[4] Billy E. Gillett, (1976), " Introduction to Operations Research", McGraw-Hill, Inc, New York.

[5] Dan Reid, R., and Nada, R.S., (2007) "Operations Management " $3^{\text {rd }}$ Edition, Wiley.

[6] Jeffery, W.H., and Chun-Lee, 1., (1992) "On Scheduling to Minimize EarlinessTardiness and Batch Delivery Cost with a Common Due Data"

[7] Kenneth, R.B., and Gary, D.S., (1990) "Sequencing with Earliness and Tardiness Penalties :a Review" Operational Research, vol.(38), No.(1), pp.2227.

[8] Kutanoglu, E., and Sabuncuoglu, I., (1999) "An Analysis of Heuristics in a Dynamic Job Shop with Weighted Tardiness Objectives" International Journal of Production research. Vol. (37), No. (1), pp.165-187.

[9] Repsalainen, A., and Morton, T.E., (1987), "Priority Rules for Job Shop with Weighted Tardiness Costs", Management Science, Vol.(33), pp 1035-1047. 


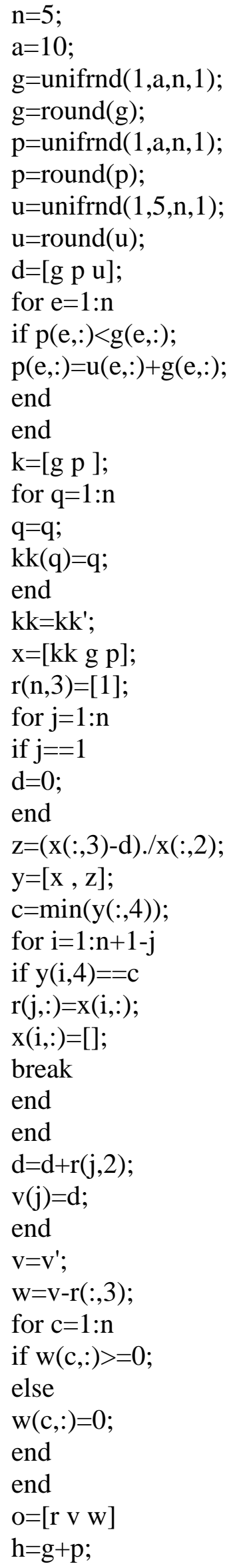




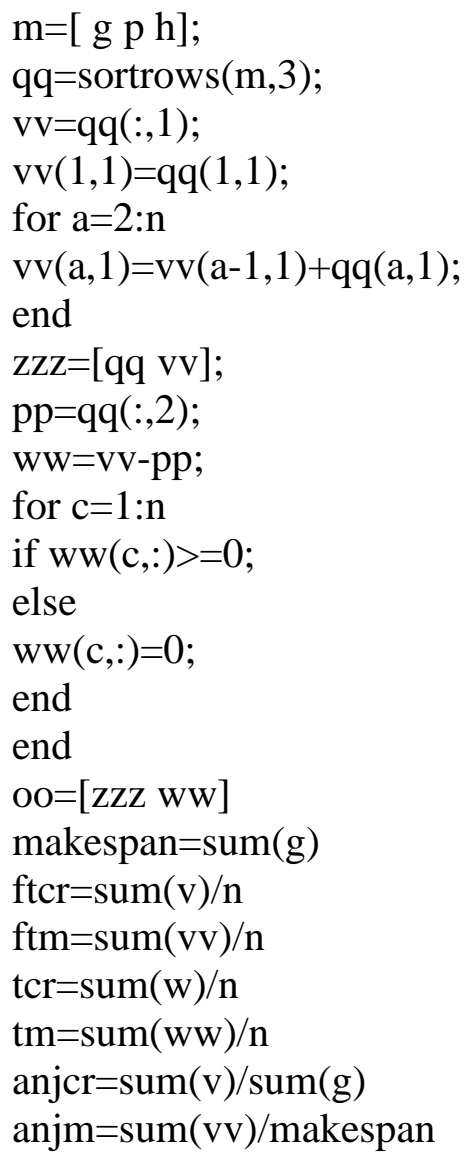

